\title{
Rheumatoid arthritis with extensive lung lesions
}

\author{
C FELLBAUM, W DOMEJ, H POPPER \\ From the Institute of Pathology, University of Graz School of Medicine, and the Department of Pneumonology, \\ Landeskrankenhaus, Graz, Austria
}

\begin{abstract}
A man of 74 who had had seropositive rheumatoid arthritis for 10 years presented with dyspnoea and reticular striation in both lung fields. At necropsy two years later there was pulmonary fibrosis with multiple rheumatoid nodules and non-specific granulomas.
\end{abstract}

Various non-specific pulmonary changes, including interstitial pneumonia, honeycomb lung, and serofibrinous pleurisy, have been reported in association with rheumatoid arthritis. ${ }^{1-5}$ Rheumatoid nodules are rare: in four studies covering 1653 patients they were found in only three cases. ${ }^{6}$ The lung nodules may precede the onset of rheumatoid arthritis by months or years, ' and their presence is not associated with the severe joint disease ${ }^{5}$ or with serological abnormalities. ${ }^{67}$ They are usually subpleural, and may be solitary or multiple. ${ }^{8}$ Sarcoid like granulomas have also been described in the lungs of patients with rheumatoid arthritis. ${ }^{4}$

We report a case of seropositive rheumatoid arthritis in which there were multiple small rheumatoid nodules and non-specific granulomas throughout the lung parenchyma.

\section{Case report}

The patient, a man of 74 years, was first admitted to the General Hospital, Graz, complaining of intermittent dyspnoea on exertion. He had had pulmonary tuberculosis $\mathbf{4 0}$ years previously, and seropositive rheumatoid arthritis for 10 years. He had also been treated conservatively for duodenal ulceration, and was known to have mild ischaemic heart disease with nodal extrasystoles.

On examination there was slight painful swelling of the interphalangeal joints of both hands, but otherwise his general condition was good. Laboratory investigations showed increased concentrations of $\alpha_{1}$ and $\alpha_{2}$ globulins and a $\gamma$ globulin concentration of $28 \mathrm{mg} / 100 \mathrm{ml}$. The erythrocyte sedimentation rate was raised to $120 \mathrm{~mm}$ in one hour (Westergren). Chest radiolography showed non-specific "fibrotic" change in the left upper lobe and reticular striation of both lungs, especially in the peripheral areas of the lower lobes (fig 1). Pulmonary function tests indicated a restrictive ventilatory defect with a vital capacity of $2 \cdot 2$ (predicted value 3.4) 1. In addition, the ratio of forced expiratory volume to

Address for reprint requests: Dr Helmut Popper, Institute of Pathology, University of Graz, Auenbruggerplatz 25, A-8036 Graz, Austria.

Accepted 12 October 1988 forced vital capacity (FEV/FVC) was only $58 \%$ of the $\underset{x}{x}$ predicted value.

His respiratory and cardiac problems were treated with theophylline, digoxin, and isosorbide dinitrate. Cortico- $>$ steroids, adrenocorticotrophin, anti-inflammatory agents, diclofenac sodium, and a short course of chloroquine phosphate were also administered. The pulmonary lesions progressed nevertheless, and the patient died in respiratory failure two years after presentation.

At necropsy the right pleural cavity was obliterated. The lungs were heavy and of firm consistency; their cut surfaces were fibrotic and greyish white to greyish red with numerous small grey subpleural and intrapulmonary nodules up to $3 \mathrm{~mm}$ in diameter. Bronchi and bronchioles were dilated. The right ventricle was hypertrophied and dilated, and there was chronic congestion of the liver, spleen, and kidneys. Only the metacarpophalangeal joint of the second finger of the right hand showed rheumatoid change.

Microscopically, almost all of the alveolar septa were

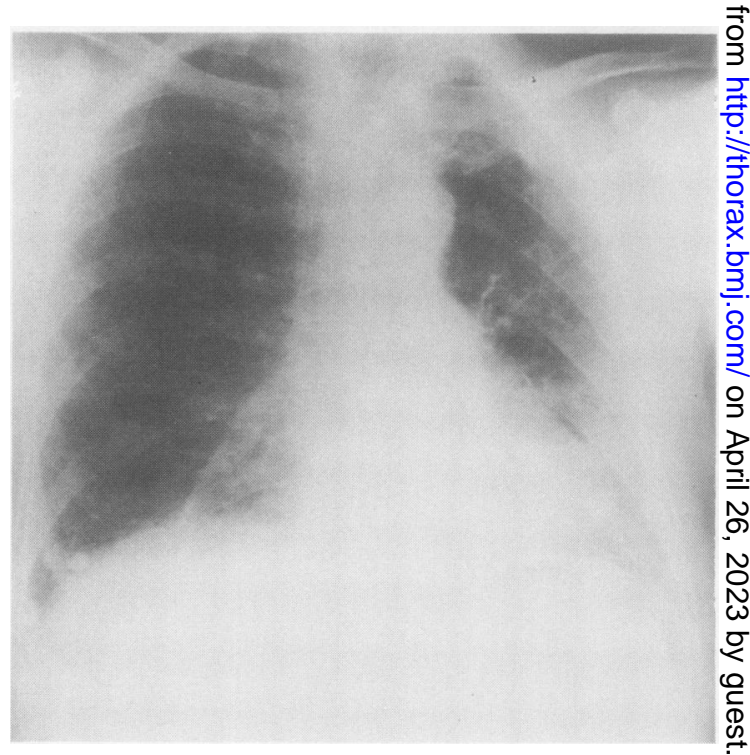

Fig 1 Chest radiograph showing changes in the left upper lobe that are thought to represent post-tuberculous fibrosis and a pea sized calcified lesion in the right lower lobe, likely to be an old tuberculous lesion. The striated and patchy shadowing in both lower lobes is attributed to the changes associated with rheumatoid arthritis. 

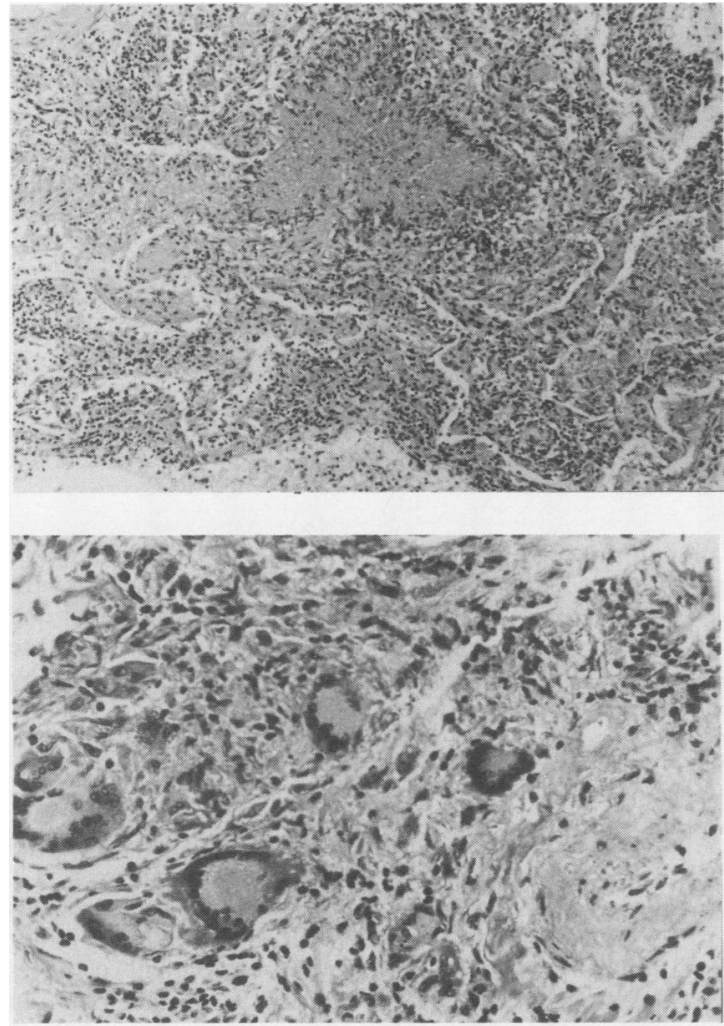

Fig 2 (a) Rheumatoid nodule showing fibrinoid necrosis and palisading histiocytes. (Haematoxylin and eosin.) (b) Perivascular granuloma consisting of histiocytes, macrophages, giant cells of Langhans and of foreign body type, and lymphocytes (Haematoxylin and eosin.)

thickened and fibrotic, and infiltrated by lymphocytes and plasma cells. Two types of granuloma, up to $3 \mathrm{~mm}$ in diameter, were present. Most were typical rheumatoid nodules, and consisted of a focus of fibrinoid necrosis surrounded by a palisade of histiocytes with scattered lymphocytes and plasma cells (fig $2 a$ ). The areas of fibrinoid necrosis were positive for IgG and the $3 \mathrm{a}$ and $5 \mathrm{a}$ components of complement by immunofluorescence. IgA and IgM were not present. In addition, there were granulomas composed of histiocytes, macrophages, giant cells of Langhans and of foreign body type, and lymphocytes (fig 2b). These latter granulomas showed different degrees of fibrosis; in some of them collagen fibres were arranged in concentric arrays around inflammatory cells.

\section{Discussion}

Pathological changes in the lung in rheumatoid arthritis include rheumatoid nodules, usual interstitial pneumonia, bronchiolitis obliterans with organising pneumonia, lym- phoid hyperplasia, and cellular interstitial infiltrates. ${ }^{4}$ Usual interstitial pneumonia may progress to a generalised interstitial fibrosis. ${ }^{4}$ In some instances the changes can be attributed to drugs: both usual interstitial pneumonia and bronchiolitis with organising pneumonia have been described as complications of penicillamine and gold treatment. $^{410}$ Lymphoid hyperplasia, lymphocytic interstitial pneumonitis, and progressive interstitial fibrosis are not specific, and may occur in other collagen disorders. The only characteristic lesion occurring in the lung in rheumatoid arthritis is the rheumatoid nodule, ${ }^{45}$ which is found in a small minority of cases. ${ }^{157}$ They are generally subpleural, varying in size from microscopic to $25 \mathrm{~mm}$ in diameter. ${ }^{24}$ Nonspecific sarcoid like granulomas have also been described, ${ }^{4}$ but whether they are a direct manifestation of rheumatoid arthritis is not certain.

In this case there were multiple rheumatoid nodules, sarcoid like granulomas, and extensive interstitial fibrosis. Other granulomatous pulmonary diseases, such as Wegener's granulomatosis, periarteritis nodosa, hypersensitivity angiitis, bronchocentric granulomatosis, Churg-Strauss syndrome, and sarcoidosis and necrotising sarcoidal granulomatosis could be excluded on morphological grounds. ${ }^{8}$ Mycobacterial and mycotic infection could also be excluded, as no organisms were detected with the Ziehl-Neelsen stain, periodic acid-Schiff stain, or silver impregnation techniques. The patient had not received gold or penicillamine treatment.

The pulmonary changes in this patient, in our opinion, can all be attributed to rheumatoid arthritis, although the extent of the lung lesions and their microscopic appearance are unusual. Possibly antigen-antibody complexes were deposited in alveolar septa, with subsequent complement activation and the formation of rheumatoid nodules and non-specific granulomas. Proliferation of fibroblasts within these lesions resulted in the production of large amounts of collagen fibres, with subsequent scarring and severe interstitial fibrosis.

\section{References}

1 Walker W, Wright V. Pulmonary Lesions and Rheumatoid Arthritis. Medicine (Baltimore) 1968;47:501-20.

2 Scadding JG. The lungs in rheumatoid arthritis. Proc $R$ Soc Med 1969;62:1-12.

3 Petty TL, Wilkins $M$. The five manifestations of rheumatoid lung. Dis Chest 1966;49:75-82.

4 Yousem SA, Colby TV, Carrington CB. Lung biopsy in rheumatoid arthritis. Am Rev Respir Dis 1985;131:770-7.

5 Patterson C, Harville W, Pierce J. Rheumatoid Lung Disease. Ann Intern Med 1965;62:685-97.

6 Eraut D, Evans J, Caplin M. Pulmonary necrobiotic nodules without rheumatoid arthritis. Br J Dis Chest 1978;72:301-6.

7 Payne C. Pulmonary manifestations of rheumatoid arthritis. $\mathrm{Br} \mathrm{J}$ Hosp Med 1984;32:192-7.

8 Katzenstein A, Askin F. Surgical pathology of non-neoplastic lung disease. Philadelphia: Saunders, 1982:139-60, 166-99.

9 Eastmond C. Diffuse alveolitis as complication of penicillamine treatment for rheumatoid arthritis. Br Med J 1976;i:1506.

10 Scott D, Bradby G, Altman T. Relationship of gold and penicillamine therapy to diffuse interstitial lung disease. Ann Rheum Dis 1981;40:136-41. 